\title{
Human Trichuriasis: Diagnostics Update
}

\author{
Beatrice Divina Barda $^{1,2}$. Jennifer Keiser ${ }^{1,2} \cdot$ Marco Albonico $^{3}$
}

Published online: 7 October 2015

(C) Springer International Publishing AG 2015

\begin{abstract}
Trichuris trichiura infection is part of the so-called neglected tropical diseases, given the little interest and resources spent in developing novel diagnostic tools and treatment to detect and fight this disease. One of the main neglected aspects of trichuriasis pertains to diagnostic methods, which are currently based on copro-parasitological methods and burdened by low sensitivity. This leads to different levels of underestimation of the real prevalence and morbidity caused by T. trichiura, in both public health and individual patient management. Only few new diagnostic methods showing good performance and affordability have been standardized and fine-tuned. Molecular-based diagnostics such as those based on the polymerase chain reaction are the main diagnostic tool for infectious diseases nowadays, but their use in parasitology is still limited owing to their high cost making them unaffordable in many countries where T. trichiura is endemic. Research and innovation are needed in order to develop new and accurate methods that are accessible for the diagnosis of this parasite and of other soiltransmitted helminths.
\end{abstract}

Keywords Trichuris trichiura . Formol-ether concentration method $\cdot$ Kato-Katz method $\cdot$ McMaster method . Mini-FLOTAC method $\cdot$ PCR $\cdot$ Diagnosis

This article is part of the Topical Collection on Topics Exploring Loa-Loa, Onchocerciasis, Hookworm, Ascaris, Trichuris

Marco Albonico

albonico@tin.it

1 Department of Medical Parasitology and Infection Biology, Swiss Tropical and Public Health Institute, Basel, Switzerland

2 University of Basel, Basel, Switzerland

3 Fondazione Ivo de Carneri, via IV Marzo 14, 10122 Torino, Italy

\section{Introduction}

Infections with the soil-transmitted helminths (STHs) Trichuris trichiura, Ascaris lumbricoides, and hookworm affect more than 800 million people worldwide [1], among which almost 500 million are infected with T. trichiura. About one billion school-aged children live in countries where the prevalence of STHs is above $20 \%$, hence at risk of acquiring an infection [1]. However, prevalence of STHs is likely to be still widely underestimated, mainly because sensitive, reliable, and accurate diagnostic methods are lacking [2••]. STH infections are part of the so-called neglected tropical diseases (NTDs), with a burden of disease of more than 5 million disability-adjusted life years (DALYs) in 2010. Of these, 0.64 million were attributable to T. trichiura [3]. Moreover, the number of years lived with disability (YLD) attributable to T. trichiura infection has been estimated as $13 \%$ of the total burden of STHs in 2010 [1].

There is little interest to invest in novel drugs and diagnostics for NTDs as these diseases, notwithstanding their wide distribution, hit mainly poor populations of middle-low income countries where there is no lucrative market. Between 2007 and 2013, US $\$ 22,418,733,255$ was spent globally for research and drug development, of which US\$6,222,526 $(<0.03 \%)$ was used for T. trichiura [4]. Data show that between 2000 and 2011, 37 out of 850 new therapeutic products (formulations, molecules, vaccines, and biological) were for NTDs, but only $1 \%$ was actually approved [5]. The lack in research and development for novel, innovative therapeutic options is flanked by scarceness in new diagnostic tools. However, diagnostic accuracy is crucial since reliable data on prevalence and intensity of infection are needed for planning future interventions. Moreover, accurate diagnostic tools are essential to assess and monitor the efficacy of treatment [6-8]. Due to the absence of a standardized procedure, clinical trials on 
drug efficacy in endemic countries have been conducted utilizing a variety of parasitological methods, including molecular techniques, to detect egg reduction rate (ERR) and cure rate (CR) in T. trichiura-infected children $[6,9 \bullet, 10 \bullet, 11 \bullet]$. The choice of the most appropriate diagnostic methods must take into account the accuracy of the method, the endemicity of T. trichiura infections, and the availability of resources including the cost of the diagnostics. In areas where STHs are endemic - mainly settings with limited resources - techniques with moderate sensitivity, like Kato-Katz, could be used for quantitative and qualitative diagnosis. On the other hand, in areas of low endemicity and those where control strategies approach to elimination and a robust surveillance system should be built up, more sensitive and specific tools are required moving from good-old direct copro-parasitological techniques toward more sophisticated albeit more expensive molecular or serological diagnostics $[12 \bullet \bullet, 13,14 \bullet]$. Although of great importance, public health is not the only drive for research: Also individual patient management requires improved diagnostics. In addition, standard operating procedures to monitor drug efficacy and early detection of drug resistance need sensitive diagnostics to alert when drug-resistant strains are still below the threshold of clinical treatment failures [15].

The aim of this review is to present the state of art of the diagnostic tools currently applied for detection of $T$. trichiura with a special focus on recently developed techniques. We discuss different approaches for diagnosis of T. trichiura infection, highlighting innovations and new fields of research.

\section{Current Diagnostic Methods Applied for T. Trichiura Infection}

One of the first evaluation of quantitative diagnosis of a Trichuris infection before and after treatment measuring egg counts and worm burden dates back in 1950 [16], and still at present the diagnosis of intestinal helminth infections relies mainly on direct examination on the presence of eggs in stool samples in a quantitative or qualitative manner. Quantitative methods have the advantage to record the number of eggs per gram (EPG) of feces, a proxy measure of intensity of infection, which is an indicator directly associated with helminth morbidity.

It is worth highlighting that STHs do not release eggs at a constant rate [17]; therefore, multiple collection of fecal samples are needed to increase the sensitivity of the methods. As stated in a recent meta-analysis on sampling and its diagnostic power for STHs and schistosomes, however, oversampling has no benefit in terms of diagnostic sensitivity, workload, and costs. Collection of multiple samples is important to better estimate prevalence and cure rates, but not for accurately calculating infection intensity and ERR. [18•]. Nikolay et al. [2••] demonstrated that preparing and analyzing more than two Kato-Kato slides from one stool sample only slightly increases the sensitivity for T. trichiura detection $(84.8 \%$ two slides vs $90.5 \%$ three slides).

Qualitative direct copro-parasitological techniques include the direct smear and formol-ether concentration techniques (Fig. 1) [19••, 20•, 21]. Used in parasitology since the 1970s, the former consists of microscopic examination of roughly $2 \mathrm{mg}$ of fresh samples on a slide on the same day of collection [22]. The formol-ether concentration method (FECM) requires processing of $1-2 \mathrm{~g}$ of the sample by adding formol $(10 \%$ formaldehyde), ether, and a centrifugation step before analyzing under the microscope a few drops of the sample pellet on a slide [21] (Fig. 1). Both techniques are not quantitative, as the amount of sample is not weighed but roughly calculated, and the dilution factor is unknown. Hence, neither method is suitable for EPG calculation. As well, direct smear and FECM are both hindered by low-moderate sensitivity $[20 \cdot 23 \bullet]$ and, moreover, being qualitative techniques, their adoption in clinical trials to test drug efficacy and in epidemiological studies is far from ideal, yet still widely used [24, 25].

Another qualitative method used for STH diagnosis is the sedimentation technique after fixation with sodium acetateacetic acid-formalin (SAF) [26]. Some authors compared SAF sedimentation with standard sedimentation technique. For the former, $2 \mathrm{~g}$ of feces was deposited in containers and diluted with $7 \mathrm{ml}$ of SAF and set for spontaneous sedimentation; after $30 \mathrm{~min}$, the sediment was placed on a slide and read under the microscope. Standard sedimentation is performed using tap water instead of SAF and letting the sample rest for $2 \mathrm{~h}$ instead of $30 \mathrm{~min}$. The two methods showed good concordance for STHs (0.7-0.8) except for T. trichiura (0.3), apparently due to mistakes in homogenization [26].

These three methods (direct thick smear, FECM, and sedimentation), despite not being quantitative, allow a broader spectrum of diagnosis of the parasites potentially present in the sample. In fact, both protozoa cysts and helminth eggs as well as nematode larvae could be detected during the same examination.

More accurate diagnosis for T. trichiura eggs is achieved by quantitative methods: Kato-Katz thick smear method, McMaster method, FLOTAC and Mini-FLOTAC techniques (Fig. 1), and bio-molecular methods.

In more detail, Kato-Katz diagnostic method has been widely used in STH (including T. trichiura) public health and drug efficacy studies because (i) it has sufficient sensitivity in areas of high/moderate endemicity, (ii) it is relatively simple, (iii) most of the materials used can be recycled and utilized for several examinations, (iv) it is currently the recommended technique by the World Health Organization [19••]. This method is performed with fresh stools and can be briefly described as follows. First, a small amount of the sample is sieved and collected into a template based on a slide. The template contains a fixed amount of sample $(41.7 \mathrm{mg})$. Subsequently, the sample is stained by pressing a layer of 

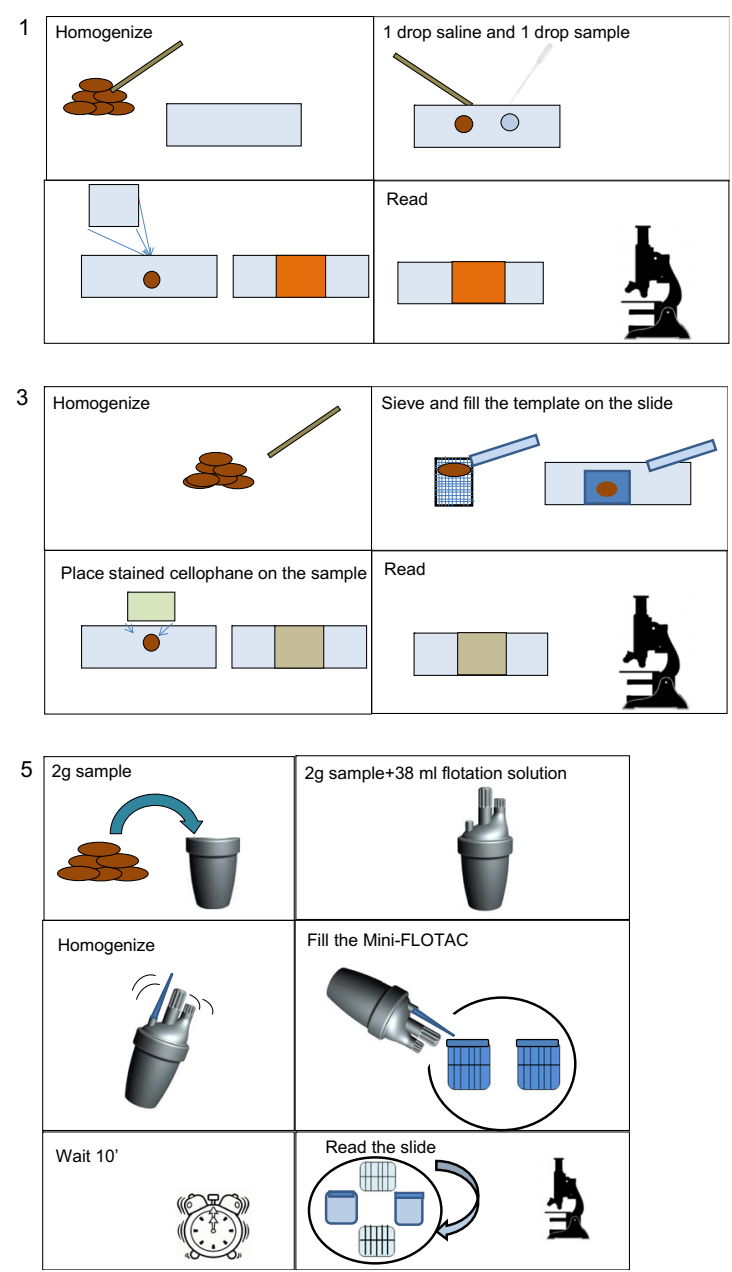
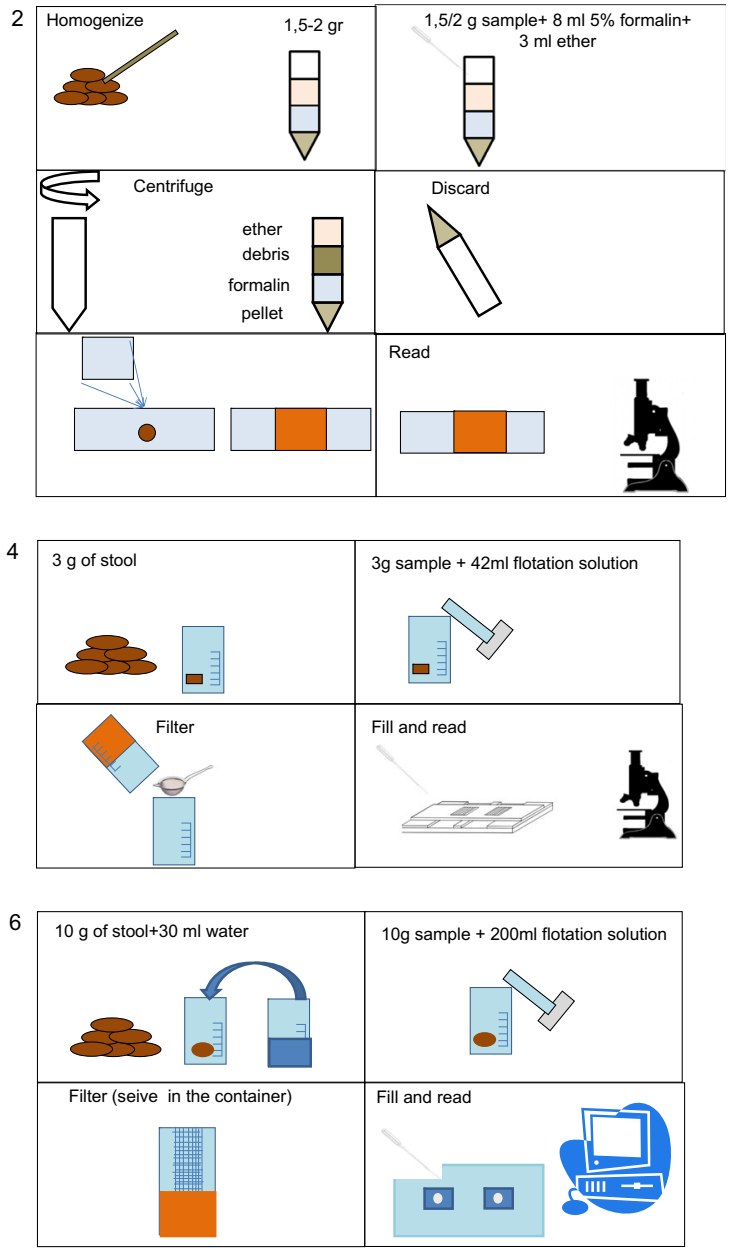

Fig. 1 Diagnostic techniques for detection of T. trichiura. (1) Direct method; (2) formol-ether concentration method; (3) Kato-Katz thick smear; (4) McMaster method; (5) Mini-FLOTAC method; (6) FekPac technique

cellophane (which has been soaked overnight in a solution of malachite green and glycerol) on the small amount collected on the slide and exposing it to the dye for at least $30 \mathrm{~min}$. The rationale of the method is that glycerol clears the fecal material while the malachite green stains the background but cannot penetrate the parasite wall, making the detection of eggs easier and clearer.

The McMaster technique is among the currently recommended direct methods for intestinal parasitic infection diagnosis $[27 \cdot \bullet$. It is widely used in veterinary medicine and is now used in human parasitology as well $[11 \bullet, 28 \bullet, 29]$. This method is based on flotation of the eggs. In brief, $3 \mathrm{~g}$ of stool is filtered and diluted (1:30) with hypersaturated saline solution; the two flotation chambers of the McMaster slide are filled with the solution and after a few minutes are read under the microscope. Hypersaturated saline allows the eggs to float and separate from debris (Fig. 1).

More than 200 studies have been conducted over the past 5 years to compare the different techniques for T. trichiura diagnosis. The unstandardized use of different techniques is due to the lack of a gold standard for diagnosis of intestinal helminth infection, including T. trichiura [28•, 29, 30]. Sayasone et al. [21] recently carried out a comparison between FECM and triplicate Kato-Katz and demonstrated that for detection of T. trichiura infection, FECM is less sensitive than Kato-Katz (73 vs $95.9 \%$, respectively). These data were also confirmed by Funk et al. [31], who found a better performance of Kato-Katz when compared to methods like formol-ethyl acetate concentration and Midi Parasep. Levecke et al. [28•] conducted a comparison between Kato-Katz and McMaster in the diagnosis of $T$. trichiura and found a slightly better although not statistically significant performance of Kato-Katz.

One of the greater limitations of direct techniques is that most require fresh stool samples, which is problematic in large studies. Alfredo Fernandez et al. [32] recently compared the diagnostic performance of Kato-Katz method on fresh versus preserved samples after 6 months of SAF fixation. The prevalence of T. trichiura in preserved samples was statistically decreased $(p<0.01)$ compared to the fresh samples [32]. Hence, the need of a diagnostic method, which allows stool preservation, is crucial. 
FLOTAC techniques are innovative methods imported from veterinary parasitology and based on flotation of the eggs with different flotation solutions that allow the separation between eggs and fecal debris [33•]. FLOTAC techniques have been tested and compared with the above mentioned methods and found to be more sensitive $[2 \bullet, 10 \bullet]$, but few limitations have been highlighted. First of all, the procedure is fairly complex and long and, just as the FECM, requires a centrifugation step, and therefore constant power supply and a large centrifuge, which are not always available in lowresource settings [10•]. Second, due to flotation solutions limits, some debris may float together with the eggs, making the egg count difficult to perform; this could be avoided by adding a further step of clarification through ether $[10 \bullet, 33 \bullet$. An advantage of the FLOTAC techniques, however, is that they can be performed on fixed samples, allowing the storage of faces for a few weeks [33•].

Mini-FLOTAC is an evolution of FLOTAC designed to be a simpler and more appropriate method to be used in resourcelimited settings, and proved to be a good alternative to KatoKatz and other direct methods (like McMaster and formolether concentration method) being both a quantitative and low-cost method (Fig. 1).

A recent study comparing different diagnostic tools for STH infections found a similar sensitivity for Kato-Katz and Mini-FLOTAC for T. trichiura detection [34•]. MiniFLOTAC is a promising alternative to currently used methods, but, because its sensitivity is similar to the that of other methods, it has not yet replaced older approaches nor fully innovated the diagnostic scenario. However, in contrast to Kato-Katz, Mini-FLOTAC uses a closed system and is therefore safer for the operator, as it does not require manipulation of fresh sample and avoids the risk of contamination [34•]. In addition, fixation with $5 \%$ formalin allows reading of the sample after at least 2 weeks after collection (even up to 30 days or longer for $T$. trichiura) with stable egg counts compared with the first examination [35•]. Preservation of samples might be helpful for field studies, including prevalence and epidemiological surveys and clinical trials, and for diagnosing STHs during Transmission Assessment Surveys for Lymphatic Filariasis $[36,37]$ where large amounts of stool samples cannot be screened immediately.

The above mentioned techniques are widely used in endemic countries for public health trials and single-patient management and diagnosis. Kato-Katz, Mc Master, and recently Mini-FLOTAC methods are also recommended in a standard protocol from WHO to monitor drug efficacy according to agreed thresholds of ERR [27••]. The main characteristics of these techniques together with some new highlights are summarized in Table 1.

When used during clinical trials, it is a good recommended practice that a subsample of the slides is randomly selected for quality control and re-examined by a different experienced laboratory technician, in order to guarantee high standard

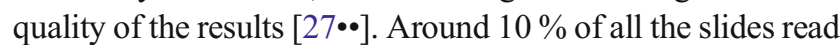
with any copro-parasitological method should be re-read; if the discordance found between the two readings is greater than $10 \%$, a third slide should be prepared and read by an independent expert. If still discordant, corrective measures should be taken (closer supervision and further training), and in the worst case, all samples should be re-analyzed. Speich et al. [38 $]$ made a systematic review of quality control procedure of Kato-Katz slides in clinical trials conducted in Pemba Island, Tanzania, during the past 3 years. The false positive rate for T. trichiura was low $(0.35 \%)$ and the false negative rate was $1.94 \%$.

\section{New Highlights}

Novel technologies sustain the development of innovative tools for the diagnosis of STHs, including T. trichiura. The new diagnostics evolve either from refinement of methods from veterinary parasitology based on remote diagnosis and telemedicine, or from detection of copro-antigens and DNAbased methods.

The FecPak ${ }^{\mathrm{G} 2}$ is a new diagnostic technique recently developed for veterinary medicine in New Zealand [39•, 40]. Similar to McMaster and Mini-FLOTAC, this method is based on the flotation of eggs using hypersaturated saline. Its innovation lays in the use of a high-tech device for the examination of the slide. Briefly, once the solution is mixed with the sample, a well is filled with the sample and read by a camera connected to a tablet with internet, which sends the image to expert parasitologists. Once the experts have analyzed the picture of the sample, they return the result to the farmer or to the technician who processed the sample and took the picture. As long as there is internet connectivity, this method might be helpful especially in remote areas, where resources are limited, equipped laboratories are not easy to reach, or trained technicians are not available. The diagnostic technique is still under development so no conclusion can yet be drawn as to whether FecPak ${ }^{\mathrm{G} 2}$ is a suitable addition to the diagnostic tools for human STH infections.

On the same track of introducing electronic devices into direct diagnosis for T. trichiura is the mobile diagnosis, which is lately gaining interest among different disciplines [41-43]. Bogoch et al. [44] used a smartphone as microscope and compared the performance of mobile and standard microscopy. Their results showed a good performance for $A$. lumbricoides and T. trichiura detection, but low sensitivity for hookworm. Moreover, images were not of good quality and often light infections were missed [44]. Bogoch and colleagues also tested a handheld light microscope for the diagnosis of STHs in comparison with standard microscope. They found that the new approach had not a better performance, showing sensitivity for all STHs of 69.4 versus $86.9 \%$ of conventional 
Table 1 Main characteristics of different diagnostic techniques for detection of T. trichiura

\begin{tabular}{|c|c|c|c|c|c|c|}
\hline & Sensitivity & Specificity & Qualitative & Quantitative & Innovation/high tech & References \\
\hline FLOTAC & +++ & +++ & +++ & ++ & +++ & {$[10 \bullet, 33 \bullet, 39 \bullet]$} \\
\hline Mini-FLOTAC & ++ & +++ & +++ & +++ & ++ & {$[20 \bullet, 34 \bullet, 35 \bullet]$} \\
\hline McMaster & ++ & +++ & +++ & ++ & + & {$[28 \bullet]$} \\
\hline Kato-Katz & ++ & +++ & +++ & +++ & + & {$[20 \bullet, 34 \bullet, 35 \bullet]$} \\
\hline Fecpak $^{\mathrm{a}}$ & ++ & +++ & ++ & ++ & +++ & {$[28 \cdot]$} \\
\hline Formol-ether concentration method & ++ & +++ & +++ & + & + & {$[9 \bullet, 10 \bullet, 28 \bullet, 31]$} \\
\hline Direct smear & + & +++ & + & & + & {$[20 \bullet]$} \\
\hline $\mathrm{PCR}^{\mathrm{a}}$ & ++++ & ++++ & ++ & ++ & ++++ & {$[51 \bullet \bullet, 53,54 \bullet]$} \\
\hline
\end{tabular}

${ }^{\text {a } U n d e r ~ e v a l u a t i o n ~}$

microscopy; sensitivity for T. trichiura detection was 54.4 versus $79.4 \%$ with conventional microscopy [45]. However, this approach worth improvement and further evaluation, as it matches new insights and technology with classical direct methods for parasitological diagnosis.

New attempts are made in order to render the stool analysis easier and user-friendly in large-scale examinations for public health purposes. Among them, many laboratories are now exploring the way of pooling samples. This approach is widely used in veterinary practice and consists of mixing a fixed weight (1 g each) of 10-60 samples together before analysis. This strategy has proved time- and cost-effective and showed a good correlation between pooled and individual egg counts for hookworm and T. trichiura infection intensity although not for A. lumbricoides egg counts [46•]. Rinaldi et al. [47] compared two diagnostic methods in the veterinary field (MiniFLOTAC vs McMaster techniques) on pooled samples for gastrointestinal strongyles and found that pooling was positively correlated with single-sample diagnosis both at baseline and at follow-up after treatment to assess ERR.

Helminth copro-antigen detection has been tested in veterinary medicine as well, but not in human diagnostics so far. Briefly, ELISA tests were performed on experimentally infected fecal samples with Trichuris vulpis eggs yielding good results compared with flotation methods. Specificity and sensitivity were acceptable compared to other ELISA assay used for intestinal infections diagnosis [48]. Further studies are needed before immunoassays can be recommended for the diagnosis of $T$. trichiura.

Molecular biology could be the new frontier of intestinal parasite diagnosis. In the past decade, the application of polymerase chain reaction (PCR) has exponentially increased, underlying its potentials in the diagnostic field [49, 50]. Being adopted mainly in human virology and bacteriology, its use has been recently extended to human parasitology [51••, 52] and even for diagnosis of T. trichiura [53]. This technique has many advantages compared to direct microscopic methods used so far: It allows a more sensitive detection of the parasite, especially at low intensity of infection; it permits to differentiate between Trichuris species with eggs of very similar morphology [53]; and it appears to have a good correlation with fecal egg counts [51••, 54•]. It is worth to underline that the amount of DNA needed to run the test is small; therefore, the collection of stool samples is not dependent on its weight, which could be a limitation when multiple direct methods are performed. Moreover, the multiplex assay allows the detection of several parasites (such as Strongyloides stercoralis, other helminthes, and protozoa) within the same test, which is a great advantage in settings where multiparasitism occurs, and their epidemiology is not clearly known. Few data are available on post-treatment reliability of PCR detection. A large study conducted with 125 children in Ecuador demonstrated that detection of $A$. lumbricoides DNA was negative 21 days after treatment with albendazole [54•]. Moreover, with DNA being a stable molecule, isolates could be stored for many years after collection, a crucial step forward in case further analyses are needed, such as the research of different pathogens or drug resistance.

Although PCR method is usually not required for T. trichiura diagnosis (since, unlike hookworm species, these worm eggs have a peculiar shape and are easily distinguished from other nematodes' eggs) [55], this sensitive method could be useful in low-prevalence settings where the aim is elimination of infection. Molecular methods like PCR and sequencing are promising tools not only for diagnostic purposes but also for their role in building up a robust surveillance system and for detection of drug-resistant strains. The main disadvantage of PCR is its high cost, as it requires not only expensive instruments but also constant power supply, specific reagents, and high cost maintenance.

T. trichiura is widely known to be less susceptible to current drugs than the other STHs [56]. This might be due to intrinsic tolerance or acquired parasitic resistance, triggered by overtreatment and the misuse of anthelminthic drugs. Strong monitoring and early detection of drug resistance rely on the development of sensitive and stable primers in order to detect resistance alleles at a threshold that is still manageable and reversible [15]. In this perspective, a few studies have 
been conducted on DNA sequencing in order to detect $\beta$ tubulin point mutations and single-nucleotide polymorphism (SNP), which might confer resistance to benzimidazole treatment [57••]. Hansen et al. [58] conducted a study on human and baboon's infection performing PCR on both adults and eggs stadia of infection and did not find SNP and mutations at codon 168,198 , or 200 of the $\beta$-tubulin gene linked to resistance. These findings are in contrast with the above mentioned study by Diawara et al. [57••], who found a significant increase of frequencies of alleles with the point mutation in codon 200 in T. trichiura specimens, although drug efficacy was not assessed.

No clear pattern of resistance has been highlighted so far in human nematodes, and data are not consistent in suggesting a clear link between SNP and reduced drug efficacy. Due to this evidence, scientific research is currently running in both directions: on one side, tracking resistance markers and new and more sensitive diagnostic tools to detect T. trichiura infection, and on the other side, testing different drugs and drug combinations against $T$. trichiura $[6,38 \cdot, 59 \bullet \bullet]$.

\section{Conclusion}

From the above review on available and novel diagnostics for T. trichiura infection, it appears that much work needs to be done before reaching an optimal level for diagnosis. Even if some standardized operating procedures have been developed, sensitive yet affordable methods are still missing. Despite many different trials conducted in this area, this issue seems not yet been solved. The lack of a diagnostic gold standard and clear guidelines, along with wide variations prompted by resource limitations in diverse settings and different research objectives, not only jeopardizes coordination among researchers and laboratory personnel but also hampers comparison of findings obtained by various studies. The WHO is currently addressing this issue through the publication of a manual on standard recommended methods for diagnosis of intestinal parasites, available also on visual aids and platforms through the web. At present, efforts are being made to develop high-tech diagnostics based on sensitive molecular-based multiplex platform that could be eventually used as point-of-care tests in countries endemic for T. trichiura and other STH infections. Special attention should be given to developing sensitive diagnostic tools to monitor drug efficacy and prevent the resistance threat against the few good anthelminthic drugs available.

\section{Compliance with Ethics Guidelines}

Conflict of Interest Beatrice Barda, Jennifer Keiser, and Marco Albonico declare that they have no conflict of interest.
Human and Animal Rights and Informed Consent This article does not contain any studies with human or animal subjects performed by any of the authors.

\section{References}

Papers of particular interest, and published recently, have been highlighted as:

- Of importance

•- Of major importance

1. Pullan RL, Smith JL, Jasrasaria R, Brooker SJ. Global numbers of infection and disease burden of soil transmitted helminth infections in 2010. Parasit Vectors. 2014;7:37.

2.• Nikolay B, Brooker SJ, Pullan RL. Sensitivity of diagnostic tests for human soil-transmitted helminth infections: a meta-analysis in the absence of a true gold standard. Int J Parasitol. 2014;44(11):76574. Comprehensive meta-analysis that underlines the gap of current diagnostic methods for STH infection and the burden of not having a "gold standard" in public health and patient management.

3. Murray CJ, Vos T, Lozano R, Naghavi M, Flaxman AD, Michaud C, et al. Disability-adjusted life years (DALYs) for 291 diseases and injuries in 21 regions, 1990-2010: a systematic analysis for the Global Burden of Disease Study 2010. Lancet. $2012 ; 380(9859)$ : 2197-223.

4. GFinder. https://gfinder.policycures.org/PublicSearchTool/results.

5. Pedrique B, Strub-Wourgaft N, Some C, Olliaro P, Trouiller P, Ford $\mathrm{N}$, et al. The drug and vaccine landscape for neglected diseases (2000-11): a systematic assessment. Lancet Glob Health. 2013;1: e371-79.

6. Speich B, Ali SM, Ame SM, Bogoch II, Alles R, Huwyler J, et al. Efficacy and safety of albendazole plus ivermectin, albendazole plus mebendazole, albendazole plus oxantel pamoate, and mebendazole alone against Trichuris trichiura and concomitant soil-transmitted helminth infections: a four-arm, randomised controlled trial. Lancet Infect Dis. 2015;15(3):277-84.A.

7. Gyorkos TW, Maheu-Giroux M, Blouin B, Saavedra L, Casapía M. Efficacy of a single dose of Albendazole for soil-transmitted helminth infections in school children of a village in Iquitos, Perú. Rev Peru Med Exp Salud Publica. 2013;30(4):601-7.

8. Namwanje H, Kabatereine NB, Olsen A. Efficacy of single and double doses of albendazole and mebendazole alone and in combination in the treatment of Trichuris trichiura in school-age children in Uganda. Trans R Soc Trop Med Hyg. 2011;105(10):586-90.

9. Speich B, Utzinger J, Marti H, Ame SM, Ali SM, Albonico M, et al. Comparison of the Kato-Katz method and ether-concentration technique for the diagnosis of soil-transmitted helminth infections in the framework of a randomised controlled trial. Eur J Clin Microbiol Infect Dis. 2014;33(5):815-22. This paper compares different techniques that are currently used in parasitology and underlines advantages and disadvantages of each method.

10. Knopp S, Speich B, Hattendorf J, Rinaldi L, Mohammed KA, Khamis IS, et al. Diagnostic accuracy of Kato-Katz and FLOTAC for assessing anthelmintic drug efficacy. PLoS Negl Trop Dis. 2011;5(4), e1036. This article assesses other techniques that were not mentioned in the previous reference, in the framework of drug efficacy.

11. Albonico M, Ame SM, Vercruysse J, Levecke B. Comparison of the Kato-Katz thick smear and McMaster egg counting techniques for monitoring drug efficacy against soil-transmitted helminths in schoolchildren on Pemba Island, Tanzania. Trans R Soc Trop Med 
Hyg. 2012;106(3):199-201. Highlights that comparison of different techniques' sensitivity is crucial in public health and in monitoring drug efficacy, especially in absence of a "true gold standard".

12.• Bergquist R, Johansen MV, Utzinger J. Diagnostic dilemmas in helminthology: what tools to use and when? Trends Parasitol. 2009;25(4):151-6. It nicely articulates the need of sensitive and specific diagnostic tools in public health helminthology when diseases approach elimination and transmission control.

13. Bergquist R, Yang GJ, Knopp S, Utzinger J, Tanner M. Surveillance and response: tools and approaches for the elimination stage of neglected tropical diseases. Acta Trop. 2015;141(Pt B): 229-34.

14. Cringoli G, Rinaldi L, Albonico M, Bergquist R, Utzinger J. Geospatial (s)tools: integration of advanced epidemiological sampling and novel diagnostics. Geospat Health. 2013;7(2):399-404. Interesting discussion on advances in epidemiological sampling, diagnostic tools and geospatial methodologies.

15. Vercruysse J, Albonico M, Behnke J, Kotze A, Prichard R, Mc Carthy J, Montresor A, Levecke B. Is anthelmintic resistance a concern for the control of human soil-transmitted helminths?. Int $\mathrm{J}$ Parasitol Drugs Drug Resist. 2011, 1-14-27.

16. Burrows RB. On the estimation of Trichuris worm burdens in patients. J Parasitol. 1950;36(3):227-31.

17. Krauth SJ, Coulibaly JT, Knopp S, Traoré M, N'Goran EK, Utzinger J. An in-depth analysis of a piece of shit: distribution of Schistosoma mansoni and hookworm eggs in human stool. PLoS Negl Trop Dis. 2012;6(12), e1969.

18. Levecke B, Brooker SJ, Knopp S, Steinmann P, Sousa-Figueiredo JC, Stothard JR, et al. Effect of sampling and diagnostic effort on the assessment of schistosomiasis and soil-transmitted helminthiasis and drug efficacy: a meta-analysis of six drug efficacy trials and one epidemiological survey. Parasitology. 2014;141(14):1826-40. Underlines the importance of stool sampling when assessing anthelminthic drug efficacy and in prevalence surveys.

19.• WHO. Bench aids for the diagnosis of intestinal parasites. Geneva, 1994. A benchmark on the classical copro-parasitological methods recommended by the WHO. It is recently being updated.

20. Barda BD, Rinaldi L, Ianniello D, Zepherine H, Salvo F, Sadutshang T, et al. Mini-FLOTAC, an innovative direct diagnostic technique for intestinal parasitic infections: experience from the field. PLoS Negl Trop Dis. 2013;7(8), e2344. First field evaluation of the Mini-FLOTAC method and its comparison with formol-ether concentration and other diagnostic techniques.

21. Sayasone S, Utzinger J, Akkhavong K, Odermatt P. Repeated stool sampling and use of multiple techniques enhance the sensitivity of helminth diagnosis: a cross-sectional survey in southern Lao People's Democratic Republic. Acta Trop. 2015;141(Pt B):315-21.

22. Unruh DH, King JE, Eaton RD, Allen JR. Parasites of dogs from Indian settlements in northwestern Canada: a survey with public health implications. Can J Comp Med. 1973;37(1):25-32.

23. Machicado JD, Marcos LA, Tello R, Canales M, Terashima A, Gotuzzo E. Diagnosis of soil-transmitted helminthiasis in an Amazonic community of Peru using multiple diagnostic techniques. Trans R Soc Trop Med Hyg. 2012;106(6):333-9. A paper from Latin American scientists on the diagnosis of soil transmitted helminths comparing different diagnostic approaches.

24. Nyantekyi L, Legesse M, Medhin G, Animut A, Tadesse K, Macias $\mathrm{C}$, et al. Community awareness of intestinal parasites and the prevalence of infection among community members of rural Abaye Deneba area. Ethiopia Asian Pac J Trop Biomed. 2014;4 Suppl 1: S152-7.

25. Mamo H. Intestinal parasitic infections among prison inmates and tobacco farm workers in Shewa Robit, north-central Ethiopia. PLoSOne. 2014;9(6), e99559.
26. Gonçalves AQ, Abellana R, Pereira-da-Silva HD, Santos I, Serra PT, Julião GR, et al. Comparison of the performance of two spontaneous sedimentation techniques for the diagnosis of human intestinal parasites in the absence of a gold standard. Acta Trop. 2014;131:63-70.

$27 . \bullet$ WHO. Assessing the efficacy of anthelminthic drugs against schistosomiasis and soil-transmitted helminthiases. Geneva: World Health Organization; 2013. WHO/HTM/NTD/PCT/2013.4. This manual recommends standard diagnostic methods for assessing efficacy of drugs against STH infection including T. trichiura.

28. Levecke B, Behnke JM, Ajjampur SSR, Albonico M, Ame SM, Charlier J, et al. A comparison of the Sensitivity and Fecal Egg Counts of McMaster Counting Technique and Kato-Katz ThickSmear methods for Soil-Transmitted Helminths. PLoS Negl Trop Dis. 2011;5(6), e1201. The first study that compares Kato-Katz and McMaster methods for diagnosis of human STH in a largescale multicentric trial.

29. Levecke B, De Wilde N, Vandenhoute E, Vercruysse J. Field validity and feasibility of four techniques for the detection of Trichuris in simians: a model for monitoring drug efficacy in public health? PLoS Negl Trop Dis. 2009;3(1), e366.

30. Albonico M, Rinaldi L, Sciascia S, Morgoglione ME, Piemonte M, Maurelli MP, et al. Comparison of three copromicroscopic methods to assess albendazole efficacy against soil-transmitted helminth infections in school-aged children on Pemba Island. Trans R Soc Trop Med Hyg. 2013;107(8):493-501.

31. Funk AL, Boisson S, Clasen T, Ensink JH. Comparison of KatoKatz, ethyl-acetate sedimentation, and Midi Parasep ${ }^{\circledR}$ in the diagnosis of hookworm, Ascaris and Trichuris infections in the context of an evaluation of rural sanitation in India. Acta Trop. 2013;126(3): 265-8.

32. Alfredo Fernández-Niño J, David Ramírez J, Consuelo López M, Inés Moncada L, Reyes P, Darío HR. Agreement of the Kato-Katz test established by the WHO with samples fixed with sodium acetate analyzed at 6 months to diagnose intestinal geohelminthes. Acta Trop. 2015;146:42-4.

33. Cringoli G, Rinaldi L, Maurelli MP, Utzinger J. FLOTAC: new multivalent techniques for qualitative and quantitative copromicroscopic diagnosis of parasites in animals and humans. Nat Protoc. 2010;5(3):503-15. The first paper that describes in detail the FLOTAC method.

34. Barda B, Cajal P, Villagran E, Cimino R, Juarez M, Krolewiecki A, et al. Mini-FLOTAC, Kato-Katz and McMaster: three methods, one goal; highlights from north Argentina. Parasit Vectors. 2014;7:271. Paper on diagnostic methods and comparison of the MiniFLOTAC vs currently used techniques.

35. Barda B, Albonico M, Ianniello D, Ame SM, Keiser J, Speich B, et al. How long can stool samples be fixed for an accurate diagnosis of soil-transmitted helminth infection using Mini-FLOTAC? PLoS Negl Trop Dis. 2015;9(4), e0003698. A study that indicates how long stool samples can be preserved for a reliable diagnosis of STH infections with Mini-FLOTAC.

36. Chu BK, Gass K, Batcho W, Ake M, Dorkenoo AM, et al. Pilot assessment of soil-transmitted helminthiasis in the context of transmission assessment surveys for lymphatic filariasis in Benin and Tonga. PLoS Negl Trop Dis. 2014;8, e2708.

37. WHO. Assessing the epidemiology of soil-transmitted helminths during a Transmission Assessment Survey in the Global Programme for the Elimination of Lymphatic Filariasis. Geneva: World Health Organization; 2015. WHO/HTM/NTD/PCT/2015.2.

38. Speich B, Ali SM, Ame SM, Albonico M, Utzinger J, Keiser J. Quality control in the diagnosis of Trichuris trichiura and Ascaris lumbricoides using the Kato-Katz technique: experience from three randomised controlled trials. Parasit Vectors. 2015;8:82. B. Highlights the importance and the accuracy of quality control using the Kato-Katz technique. 
39. Bosco A, Rinaldi L, Maurelli MP, Musella V, Coles GC, Cringoli G. The comparison of FLOTAC, FECPAK and McMaster techniques for nematode egg counts in cattle. Acta Parasitol. 2014;59(4):6258 . Information from veterinary medicine comparing new diagnostic techniques with currently used methods.

40. Godber OF, Phythian CJ, Bosco A, Ianniello D, Coles G, Rinaldi L, et al. A comparison of the FECPAK and Mini-FLOTAC faecal egg counting techniques. Vet Parasitol. 2015;207(3-4):342-5.

41. Nasi G, Cucciniello M, Guerrazzi C. The role of mobile technologies in health care processes: the case of cancer supportive care. J Med Internet Res. 2015;17(2), e26.

42. Coates SJ, Kvedar J, Granstein RD. Teledermatology: from historical perspective to emerging techniques of the modern era: part I: History, rationale, and current practice. J Am Acad Dermatol. 2015 ;72(4):563-74; quiz 575-6. A).

43. Coates SJ, Kvedar J, Granstein RD. Teledermatology: from historical perspective to emerging techniques of the modern era: part II: Emerging technologies in teledermatology, limitations and future directions. J Am Acad Dermatol. 2015;72(4):577-86; quiz 587-8. B).

44. Bogoch II, Andrews JR, Speich B, Utzinger J, Ame SM, Ali SM, et al. Mobile phone microscopy for the diagnosis of soil-transmitted helminth infections: a proof-of-concept study. Am J Trop Med Hyg. 2013;88(4):626-9.

45. Bogoch II, Andrews JR, Speich B, Ame SM, Ali SM, Stothard JR, et al. Quantitative evaluation of a handheld light microscope for field diagnosis of soil-transmitted helminth infection. Am J Trop Med Hyg. 2014;91(6):1138-41.

46. Mekonnen Z, Meka S, Ayana M, Bogers J, Vercruysse J, Levecke B. Comparison of individual and pooled stool samples for the assessment of soil-transmitted helminth infection intensity and drug efficacy. PLoS Negl Trop Dis. 2013;7(5), e2189. The first paper from the field assessing the performance of pooling of samples for STH diagnosis and monitoring drug efficacy.

47. Rinaldi L, Levecke B, Bosco A, Ianniello D, Pepe P, Charlier J, et al. Comparison of individual and pooled faecal samples in sheep for the assessment of gastrointestinal strongyle infection intensity and anthelmintic drug efficacy using McMaster and MiniFLOTAC. Vet Parasitol. 2014;205(1-2):216-23.

48. Dopchiz MC, Lavallén CM, Bongiovanni R, Gonzalez PV, Elissondo C, Yannarella F, et al. Endoparasitic infections in dogs from rural areas in the Lobos District, Buenos Aires province, Argentina. Rev Bras Parasitol Vet. 2013;22(1):92-7.

49. Espy MJ, Uhl JR, Sloan LM, Buckwalter SP, Jones MF, Vetter EA, et al. Real-time PCR in clinical microbiology: applications for routine laboratory testing. Clin Microbiol Rev. 2006;19(1):165-256.
50. Klein D. Quantification using real-time PCR technology: applications and limitations. Trends Mol Med. 2002;8(6):257-60.

$51 . \bullet$ Verweji JJ, Stensvold CR. Molecular testing for clinical diagnosis and epidemiological investigations of intestinal parasitic infections. Clin Microbiol Rev. 2014;27(2):371-418. Molecular biology method ( PCR) as a sensitive diagnostic tool for intestinal parasitic infections.

52. Taniuchi M, Verweij JJ, Noor Z, Sobuz SU, Lieshout L, et al. High throughput multiplex PCR and probe-based detection with Luminex beads for seven intestinal parasites. Am J Trop Med Hyg. 2011;84:332-7.

53. Liu GH, Zhou W, Nisbet AJ, Xu MJ, Zhou DH, Zhao GH, et al. Characterization of Trichuris trichiura from humans and T. suis from pigs in China using internal transcribed spacers of nuclear ribosomal DNA. J Helminthol. 2014;88(1):64-8.

54. Mejia R, Vicuña Y, Broncano N, Sandoval C, Vaca M, Chico M, et al. A Novel, Multi-Parallel Real-time polymerase chain reaction approach for eight gastrointestinal parasites provides improved diagnostic capabilities to resource-limited at-risk populations. Am J Trop Med Hyg. 2013;88(6):1041-7. Description of a novel multiplex qPCR method for diagnosis of intestinal parasitic infections including $T$. trichiura and correlation with egg counts.

55. Demeler J, Ramünke S, Wolken S, Ianiello D, Rinaldi L, Gahutu $\mathrm{JB}$, et al. Discrimination of gastrointestinal nematode eggs from crude fecal egg preparations by inhibitor-resistant conventional and real-time PCR. PLoS ONE. 2013;8(4), e61285.

56. Keiser J, Utzinger J. Efficacy of current drugs against soiltransmitted helminth infections: systematic review and meta-analysis. JAMA. 2008;299(16):1937-48.

57.• Diawara A, Schwenkenbecher JM, Kaplan RM, Prichard RK. Molecular and biological diagnostic tests for monitoring benzimidazole resistance in human soil-transmitted helminths. Am J Trop Med Hyg. 2013;88(6):1052-61. One of the most authoritative papers that explores the molecular markers for benzimidazole resistance in human $S T H$.

58. Hansen TV, Thamsborg SM, Olsen A, Prichard RK, Nejsum P. Genetic variations in the beta-tubulin gene and the internal transcribed spacer 2 region of Trichuris species from man and baboons. Parasit Vectors. 2013;6:236.

59.• Savioli L. Preventive anthelmintic chemotherapy - expanding the armamentarium. N Engl J Med. 2014;370(7):665-6. Authoritative editorial on the need of testing and developing new anthelminthic drug combinations using the appropriate standard protocols and diagnostic tools. 